\title{
Asbestos induced pericardial effusion and constrictive pericarditis
}

\author{
D Davies, M I J Andrews, J S P Jones
}

\begin{abstract}
The number of disorders attributable to asbestos exposure has increased gradually over the years. The latest to be recorded is pericardial effusion and constrictive pericarditis, and three cases are reported here. A man with bilateral pleural thickening and plaques developed acute pericarditis and an effusion and was treated by pericardiectomy. Two men died from constrictive pericarditis associated with bilateral pleural effusions and diffuse pleural thickening. The pericardium showed nonspecific fibrous thickening. All had been occupationally exposed to asbestos. In the fatal cases the lungs contained amphibole fibres, in keeping with a modest degree of occupational exposure. Asbestos produces progressive fibrosis of the pericardium that is similar to diffuse pleural thickening and may be fatal. Both conditions may develop after relatively short or light exposure.
\end{abstract}

City Hospital,

D Davies

J S P Jones

St Mary's Hospital Portsmouth PO3 6AD $M$ I J Andrews

Reprint requests to Professor Jones

Accepted 21 February 1991

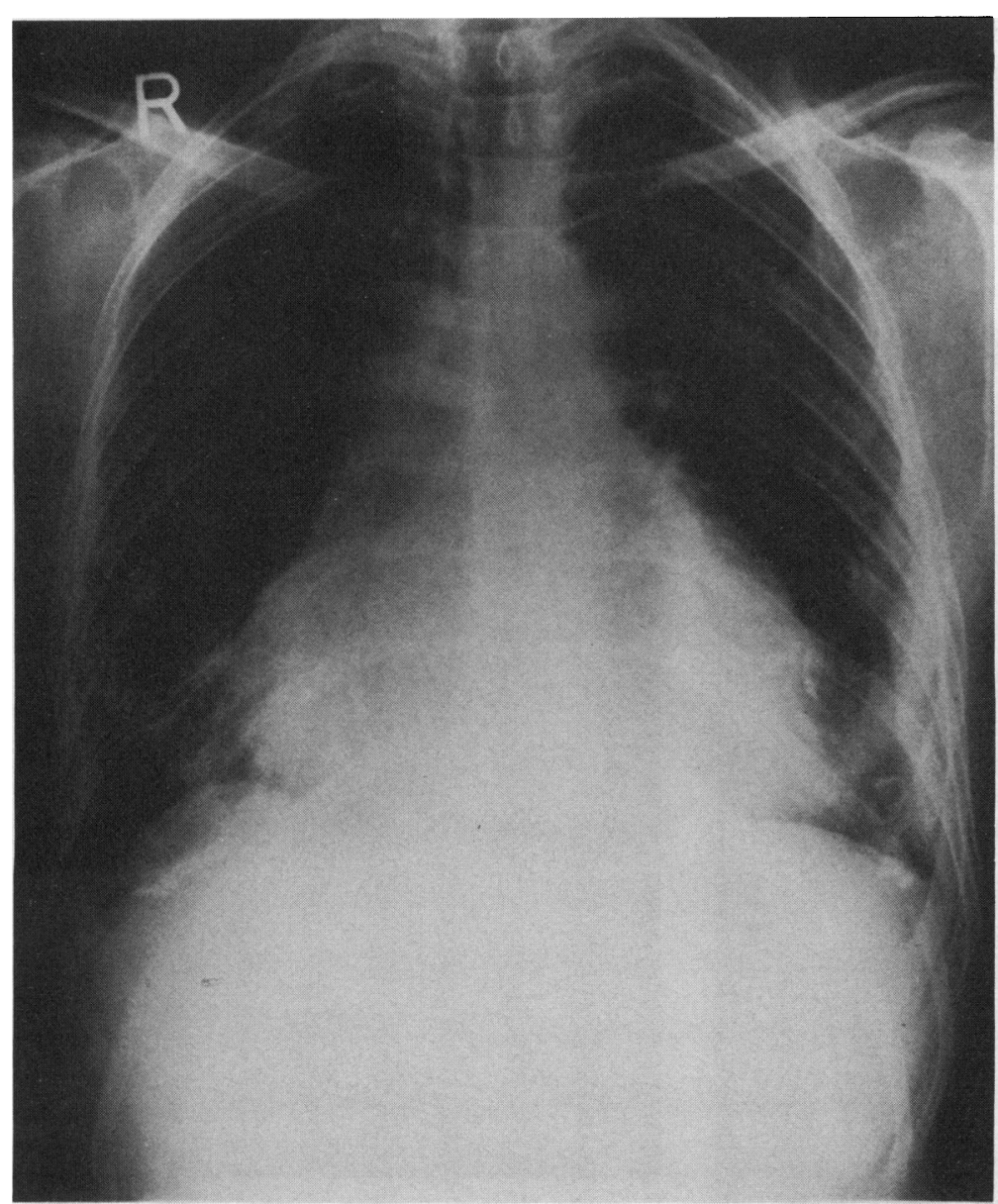

Figure 1 Chest radiograph from case 1 showing an enlarged cardiac shadow, calcified plaques and diffuse bilateral pleural thickening.
The association between asbestos exposure and diffuse lung fibrosis was established by 1930 but the recognition of other disorders caused by asbestos came slowly. The association with lung cancer was recognised by 1955 and with mesothelioma in 1960 . At about the same time the association between asbestos exposure and hyaline pleural plaques was described $^{12}$ and soon afterwards asbestos induced pleural effusions were reported, ${ }^{3}$ some of which cleared while others left considerable pleural thickening. Diffuse pleural thickening without preceding effusions ${ }^{45}$ was recognised and bilateral diffuse pleural thickening became a prescribed disease in 1985 . Another variant is visceral pleural plaque formation; this tends to cause rounded atelectasis, which mimics a tumour. ${ }^{6}$

Calcified plaques are sometimes seen on the heart border. Nearly all are in the mediastinal pleura, though occasional plaques occur in the parietal pericardium. ${ }^{7} \mathrm{~A}$ few asbestos associated primary mesotheliomas of the pericardium have been reported. ${ }^{89}$ Pleural mesotheliomas often invade the pericardium and may cause constrictive pericarditis. Two cases of asbestos associated non-malignant constrictive pericarditis have been published. ${ }^{10}{ }^{11} \mathrm{We}$ present a further three.

\section{Case reports}

\section{PATIENT 1}

In November 1983 a man of 43 was admitted to hospital with precordial pain of 12 hours' duration. No abnormality was found by clinical examination but he had neutrophilia and a raised erythrocyte sedimentation rate. The electrocardiogram showed changes of acute pericarditis. Serial cardiac enzymes were normal. Radiographs showed diffuse bilateral pleural thickening, calcified plaques, and a normal heart shadow. The pain eased and he was discharged after three days with a diagnosis of pericarditis of undetermined cause.

Fresh precordial pain led to his readmission a month later. He was febrile, the venous pressure in the neck was $3 \mathrm{~cm}$ and he had a pericardial rub. The chest radiograph showed an enlarged cardiac shadow and -unchanged pleural shadowing (fig 1). Echocardiography showed a moderate effusion.

The tuberculin skin test and blood culture gave negative results. Concentrations of cardiac enzymes, serum electrolytes, glucose, and complement were normal. Autoantibodies and antibodies against viruses, psittacosis, and coxiella were not found. The antistreptolysin titre was normal and viruses were not isolated from throat swabs or stools. 
A thoracotomy was carried out. Pleural plaques were present in the parietal layer of the lower chest wall and diaphragm. There was also diffuse fibrous thickening of the pleura and pericardium. A partial pericardiectomy was carried out.

Histologically the pleural plaques showed characteristic bundles of collagen arranged in a "basket weave" pattern. The thickened pericardium and pleura showed a similar basket weave pattern with hyalinisation and moderate lymphocytic infiltration. In addition, the pericardium showed a fibrinous exudate on its surface (fig 2). Examination of the very scanty peripheral fragments of lung tissue that were adherent to the thickened pleura showed occasional asbestos bodies of classical appearance but no asbestosis. The patient made a good recovery and there has been no progression of the pleural thickening.

Occupational history Heavy exposure to asbestos of unknown type had occurred from the age of 18 to 20 , when he worked as a lagger.

\section{PATIENT 2}

In 1982 a man aged 64 years complained of dizziness and was found to be hypertensive. No other abnormality was detected and an electrocardiogram was normal. A chest radiograph showed calcified plaques on both diaphragms. His blood pressure was controlled with hydrochlorothiazide and triamterene.

In January 1984 he developed chest tightness, a dry cough, and shortness of breath. When seen in hospital he had tachycardia, slightly enlarged liver and normal blood pressure. An electrocardiogram showed low voltage and $T$ wave inversion in leads $1,2,3, A V F$, and $\mathrm{V}$ 4-6. The chest radiograph showed a large heart and clear lung fields. Liver function tests gave abnormal results. He was considered to have had a myocardial infarction with secon-

Figure 2 Microscopic appearance of the visceral pericardium (case 1) showing hyalinised "basket weave" thickening, with a zone of lymphocytes adjacent to the myocardium and $a$ fibrinous exudate on the surface. dary congestion of the liver. Hypotensive drugs were discontinued.

He was readmitted to hospital in June 1984 because of increased shortness of breath. He had distended neck veins, a large liver, oedema up to the thighs, and a large right pleural effusion. An echocardiogram was considered to show congestive cardiomyopathy. Aspiration of the pleural effusion produced 2 litres of straw coloured pleural fluid, which formed a clot containing lymphocytes and mesothelial cells. The oedema decreased with diuretic treatment. Over the next year he remained short of breath but with little oedema. In 1985 he became more oedematous and the liver was enlarged to $5 \mathrm{~cm}$ despite large doses of diuretics. In October he had gross oedema, distended neck veins, and bilateral pleural rubs. A chest radiograph showed a large effusion on the right and a moderate effusion on the left. Repeated aspirations of the effusion on the right produced blood stained fluid with a high protein content containing red cells, lymphocytes, and a few mesothelial cells. Pleural biopsy specimens showed dense fibrous tissue only. He continued to deteriorate, with increased shortness of breath, gross neck vein distension, and large bilateral pleural effusions, and he died in January 1986.

Necropsy showed a rigid, constrictive pericardium resulting from dense hyaline thickening of both visceral and parietal layers (fig 3). The former was up to $0.2 \mathrm{~cm}$ in diameter and the latter up to $0.5 \mathrm{~cm}$. Microscopically, the pericardium varied from acellular collagenous areas to cellular fibroblastic tissue accompanied by a non-specific inflammatory reaction (fig 4). Fibrin was present on the surface. No evidence of neoplasm was seen. The pleura cavities contained $800 \mathrm{ml}$ of blood stained effusion on the right side and a similar $700 \mathrm{ml}$ effusion on the left. Calcified hyalinised pleural plaques were present on the diaphragmatic pleurae of both sides. The right lung showed

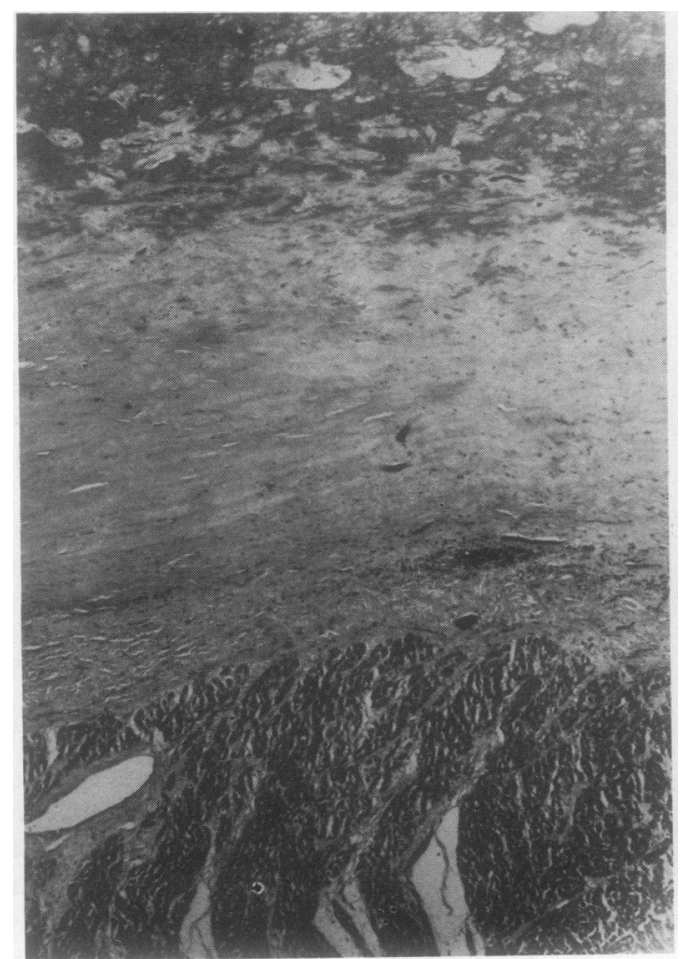

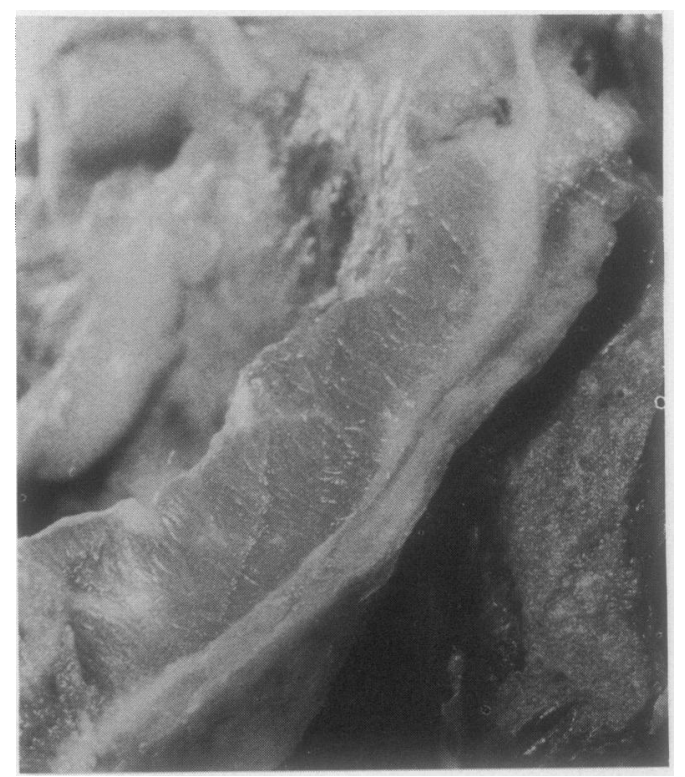

Figure 3 Hyaline thickening of visceral and parietal layers of pericardium, which have become partially fused together (case 2). 
Figure 4 Microscopic appearance of the thickened pericaridum (case 2) showing collagenous bundles of varying cellularity. edge of photograph. Myocardial fibres at lower

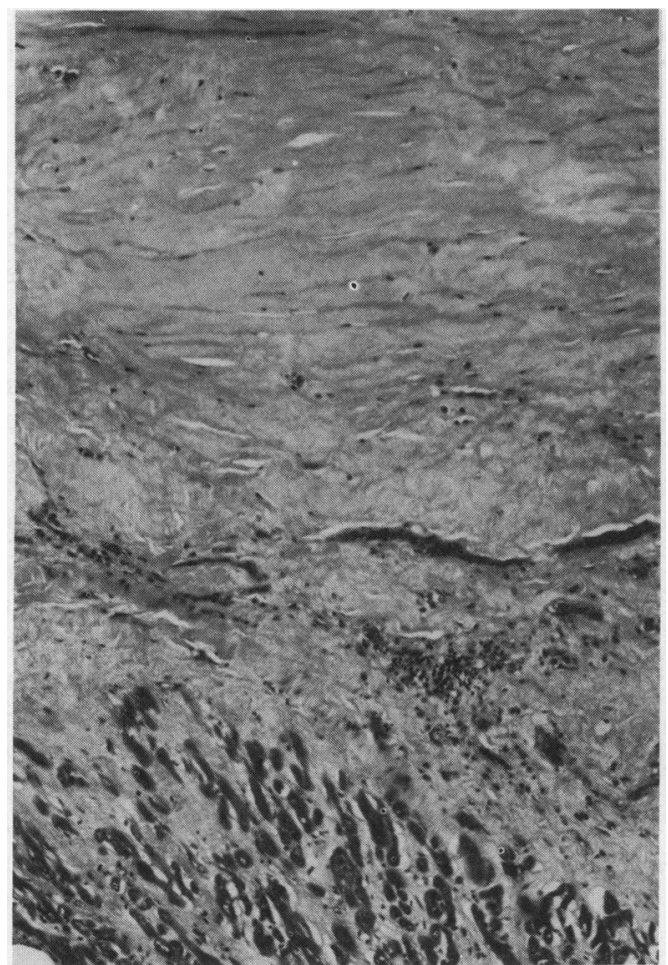

Figure 5 Diffuse fibrous thickening of visceral pleura (case 2). lung tissue showed a minor degree of asbestosis with patchy interstitial fibrosis, and asbestos bodies of classical appearance. Mineral analysis of lung tissue at the Medical Research Council unit at Penarth, using electron microscopy and energy dispersive $x$ ray analysis, showed 3.6 million fibres of crocidolite and 22 million fibres of amosite per gram of dry lung.

Occupational history In his youth this patient had served in the Merchant Navy and at the end of the war he became a marine engine fitter, working in a naval dockyard. He had been exposed to asbestos intermittently over many years until his retirement in 1982.

\section{PATIENT 3}

In July 1976 a man aged 43 years complained of weight loss and shortness of breath. Radiographs showed extensive pleural thickening over the periphery and base of the right lung and a large left pleural effusion, which was found to be blood stained. He remained unwell and short of breath. He gradually developed signs of right heart failure and the amount of pleural shadowing increased on both sides. $\mathrm{He}$ died from heart failure in 1980 at the age of 47 years.

Necropsy showed severe pericardial restriction with fusion of the visceral and parietal layers to form a diffuse dense fibrous encapsulation of the heart up to $0.9 \mathrm{~cm}$ across. The pleura showed diffuse fibrous thickening consisting of white compact hyalinised connective tissue up to $4 \mathrm{~cm}$ in diameter. In places the visceral and parietal layers of the pleura were fused, while in others a haemorrhagic effusion was present in the pleural space. Microscopically the pericardial and pleural tissues were of similar appearance, showing dense hyaline thickening of acellular collagenous material (fig 6). Capillary blood vessels were present, but there was no evidence of active inflammation. No neoplasm was present. The lung tissue showed no evidence of asbestosisthat is, no generalised fibrosis and only scanty asbestos bodies were detected by light microscopy. Mineral analysis of the lung tissue by the MRC Unit, Penarth, using electron microscopy and energy dispersive $x$ ray analysis ${ }^{12}$ showed: 2.5 million fibres of crocidolite, 0.9 million fibres of amosite, and 14.2 million fibres of chrysolite per gram of dry lung.

Occupational history In 1963 and 1964 the patient worked alongside a man who mixed an asbestos compound while casting lead grids. In 1971 he spent several months making car batteries. For this he melted bitumen in a large mixer and tipped in bags of asbestos and mica, which were then stirred in. The mixture was then poured into moulds.

diffuse thickening of the visceral pleura up to $0.2 \mathrm{~cm}$ in diameter (fig 5). The parietal pleura showed hyaline thickening up to $1 \mathrm{~cm}$. The left lung showed a lesser degree of diffuse fibrous thickening and a fibrinous deposit on the visceral pleural surface. No evidence of pleural neoplasm was present and the microscopic appearances were similar to those of the pericardium. Microscopic examination of the

\section{Discussion}

The three men had been occupationally exposed to asbestos, but by comparison with life long insulation workers, for example, their exposure was not heavy. The asbestos content with a relatively modest degree of occupational exposure to amphibole fibres. Patient 1 had of the lungs in two of the cases is in keeping 


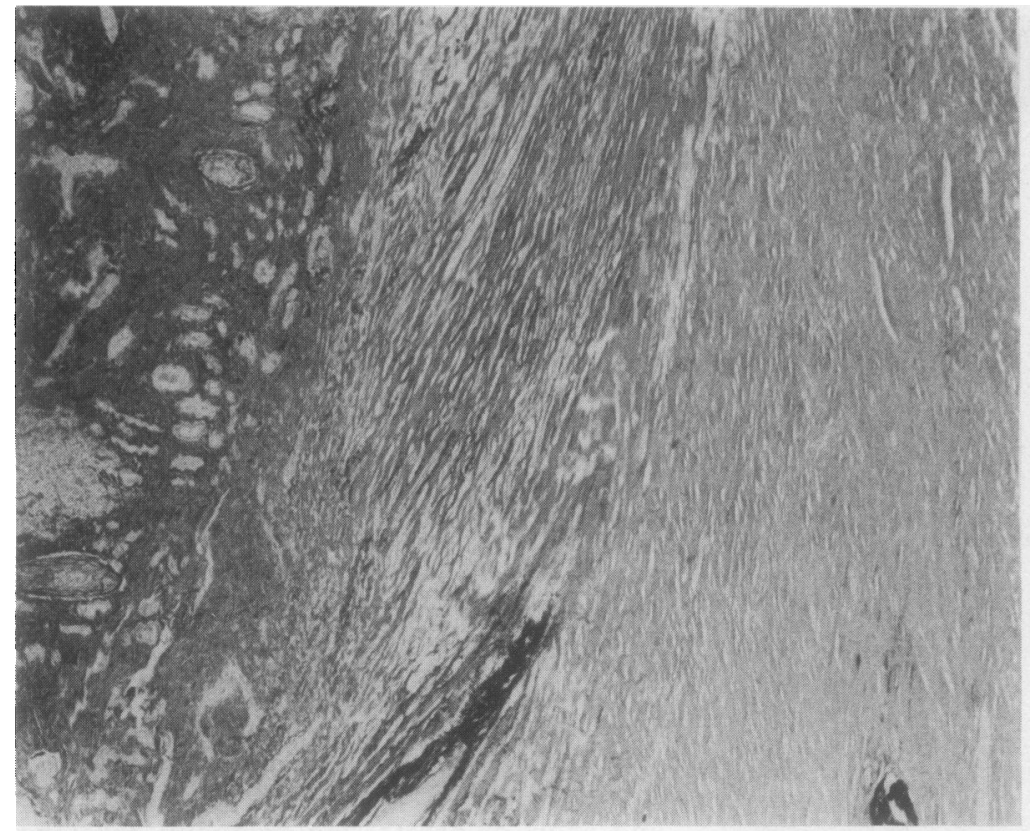

Figure 6 Acellular collagen showing "basket weave" pattern on pleural surface, with identical appearance on pericardial surface (case 3).

heavy exposure for two years to asbestos of unknown type. The exposure in patient 2 was spread over 25 years or more, but it was probably very intermittent. Patient 3 had fairly light exposure for about two years and heavy exposure for less than a year, the latter almost certainly to crocidolite. It is well recognised that relatively light exposures to amphibole asbestos may produce benign pleural fibrosis. ${ }^{13}$ Asbestosis requires heavier exposures.

All three patients had asbestos induced pleural effusions and diffuse pleural fibrosis. Patient 1 developed acute pericarditis superimposed on bilateral diffuse pleural thickening of unknown duration, which had not changed during five years of subsequent observation. The pericardial effusion appeared 23 years after his last exposure to asbestos. This is much longer than the usual interval for asbestos induced pleural effusions. The pericarditis became fibrinous, and considerable fibrous thickening developed over six weeks. He would probably have developed progressive constrictive pericarditis but for the pericardiectomy.

We believe that patient 2 began with a pericardial effusion, and the only preceding asbestos related condition was parietal pleural plaques. The fluid decreased quickly and he developed a unilateral pleural effusion some five months later. The extensive oedema and liver congestion at that time indicates that the pericardial constriction was well advanced. Bilateral pleural effusions developed 10 months after the onset of illness and persisted until the patient died from constrictive pericardial disease. The minor degree of asbestosis found at necropsy did not contribute materially to his death. The interval from first exposure to asbestos to the development of pericardial and pleural effusions was again long-almost 40 years-but the interval after his last exposure was probably fairly short. The time sequence in case 3 is less clear. The patient had established diffuse pleural thickening on the right side at the time when the left pleural effusion was detected. Whether he ever had a pericardial effusion or whether the pericardial thickening developed on its own is unknown. Probably an effusion was present at some stage. The interval between the last asbestos exposure and the onset of the pleural effusion was fairly typical at five years.

It has been suggested that the structures lining the body cavities-pleura, peritoneum, pericardium, and tunica vaginalis testis-have an identical disease pattern in response to common causative agents. ${ }^{14}$ Asbestos induced malignant mesothelioma is such an example. These three cases of diffuse pericardial fibrosis, arising in patients who had been exposed to asbestos, are presented in support of this thesis. They illustrate the way in which the pericardium has produced a pathological response identical to that of the pleura.

We thank Mr W E Morgan for permission to publish the details about patient 1 and $\mathrm{Dr} D$ Colin-Jones for the details about patient 2; Dr T C Williams and Dr R M Whittington, HM Coroner, for permission to publish the details about patient 3; Professor F D Pooley, Dr A R Gibbs, and Mr D M Griffiths for the mineral analyses; Mr Keith Miller for his technical expertise, and Mrs Valerie Bolton for the preparation of the manuscript.

1 Kiviluoto R. Pleural calcification as a roentgenologic sign of non-occupational evidence of anthophyllite asbestosis. Acta Radiol 1960;194(suppl):1-67.

2 Meurman L. Asbestos bodies and pleural plaques in a Finnish series of autopsy cases. Acta Pathol Microbiol Immunol Scand 1966;suppl 181.

3 Eisenstadt NB. Asbestos pleurisy. Dis Chest 1964;46:78-81.

4 Sheers. G, Templeton AR. Effects of asbestos in dockyard workers. Br Med J 1968;3:574-9.

5 Wright PH, Hanson A, Kreel L, Capel LH. Respiratory function changes after asbestos pleurisy. Thorax 1980; 35:31-6.

6 Mintzer RA, Gore RM, Vogelzang RL, et al. Rounded atelectasis and its association with asbestos-induced pleural disease. Radiology 1981;139:567-70.

7 Fletcher DE, Edge JR. The early radiological changes in pulmonary and pleural asbestosis. Clin Radiol 1970;21: $.355-65$.

8 Kahn EI, Rohl A, Barrett EW, Suzuki Y. Primary pericardial mesothelioma following exposure to asbestos. Environ Res 1980;23:270-81.

9 Beck B, Konetze G, Ludwig V, Rothig W, Sturm W. Malignant pericardial mesothelioma and asbestos exposure: a case report. Am J Ind Med 1982;3:149-59.

10 Fischbein $L$, Namade $M$, Sachs $R N$, Robineau $M$, Lanfranchi J. Chronic Constrictive Pericarditis associated with asbestosis. Chest 1988;94:646-7.

11 Pope AR, Sokolowski JW, Spirn I. Constrictive pericarditis Chest 1989;95:1172.

12 Pooley FD, Clark NJ. A comparison of fibre dimensions in chrysotile, crocidolite and amosite particles from samples of airborne dust and from post-mortem lung tissue specimens. In: Wagner JC, ed. Biological effects of mineral fibres. Lyon: International Agency for Research on Cancer, 1980.79-86. (IARC scientific publications No 30; INSERM symposia series Vol 92.)

13 Stephens M, Gibbs AR, Pooley FD, Wagner JC. Asbesto induced diffuse pleural fibrosis. Thorax 1987;42:583-8.

14 Jones JSP. Pathology of the mesothelium. Berlin: Springer, 1987:237-43. 